\title{
COMPETENCIAS EN INGENIERÍA Y EFICACIA INSTITUCIONAL
}

\author{
Roberto Giordano-Lerena ${ }^{1}$, Sandra Cirimelo²
}

${ }^{1}$ Decano y docente titular, Facultad de Ingeniería de la Universidad FASTA, Argentina. Presidente de Relaciones Interinstitucionales e Internacionales del Consejo Federal de Decanos de Ingeniería de Argentina Confedi Correo electrónico: rogiord@ufasta.edu.ar

${ }^{2}$ Secretaria Académica y Profesora Titular, Facultad de Ingeniería, Universidad FASTA, Argentina

Recibido: 15 de agosto del 2013. Aprobado: 30 de septiembre del 2013.

Cómo citar este artículo: R. Giordano-Lerena y S. Cirimelo, “Competencias en ingeniería y eficacia institucional”. Ingeniería Solidaria, Vol. 9, No. 16, pp. 119-127, Dic., 2013.

Resumen. Se exponen en este trabajo las competencias básicas de egreso esperadas para el ingeniero argentino, consensuadas por el Consejo Federal de Decanos de Ingeniería de Argentina, como una expresión de la meta respecto a las expectativas de formación de los egresados para las escuelas de ingeniería de ese país. Además, se expone la primera experiencia de medición de la efectividad institucional en la formación de ingenieros realizada en la Argentina, a partir del relevamiento de la percepción de los egresados respecto de las competencias genéricas buscadas. Todo esto con el propósito de dar a conocer la expresión de tales competencias y compartir la experiencia desarrollada, a efectos de promover este tipo de proyectos institucionales. En el desarrollo del trabajo, los autores reflexionan sobre la concepción actual de la sociedad respecto del egresado universitario y la consecuente necesidad de las universidades de ser efectivas en ese fin y de saber, además, cuán efectivas resultan en cuanto a dotar de competencias a sus profesionales. Finalmente, se concluye en lo valioso de la experiencia y se señala que el análisis del grado de percepción del nivel de desarrollo de estas competencias por parte de los graduados contribuye al análisis y diagnóstico institucional, y constituye un insumo significativo para el análisis, diseño e implementación procesos de gestión y cambio.

Palabras clave: competencias en ingeniería, educación en ingeniería, eficacia institucional, medición de la eficacia institucional.

\section{ENGINEERING COMPETENCIES AND INSTITUTIONAL EFFECTIVENESS}

Abstract. This study presents the basic competencies expected of a graduate engineer in Argentina, as agreed by the Consejo Federal de Decanos de Ingeniería de Argentina, setting a benchmark for the education of graduates in the country's engineering schools. Also presented here is the first attempt in Argentina at measuring how effective an institution is in educating engineers, based on a survey of graduates' perceptions about the generic competencies that are sought. The study is conducted to show how these competencies are manifested and the experience is shared in order to promote these types of institutional projects. In carrying out this study, the authors reflect about society's current conception of university graduates and the resulting need for universities to effectively fulfill expectations, and also to know how effective they have been in providing competencies to their students. Finally, the study concludes that this experience has been a valuable one and highlights that the graduates' perceptions of the degree to which they have developed the competencies contributes to the institutional analysis and assessment, and represents an important input for analysis, design and implementation of management and change processes.

Keywords: engineering competencies, engineering education, institutional effectiveness, measurement of institutional effectiveness.

\section{COMPETÊNCIAS EM ENGENHARIA E EFICÁCIA INSTITUCIONAL}

Resumo. Expõem-se neste trabalho as competências básicas esperadas para o engenheiro argentino ao se formar, acordadas pelo Conselho Federal de Decanos de Engenharia da Argentina, como uma expressão da meta a respeito das expectativas de formação dos formandos para as escolas de engenharia desse país. Além disso, expõe-se a primeira experiência de medição da efetividade institucional na formação de engenheiros realizada na Argentina, a partir do relevamento da percepção dos formandos sobre as competências genéricas buscadas. Tudo isso com o propósito de dar a conhecer a expressão dessas competências e compartilhar a experiência desenvolvida, a fim de promover esse tipo de projetos institucionais. No desenvolvimento do trabalho, os autores refletiram sobre a concepção atual da sociedade a respeito do formado universitário e a consequente necessidade das universidades de ser efetivas nesse fim e de saber, além disso, quão efetivas resultam quanto a dotar de competências a seus profissionais. Finalmente, conclui-se no valioso da experiência e sinaliza-se que a análise do grau de percepção do nível de desenvolvimento dessas competências por parte dos formados contribui para a análise e diagnóstico institucional, e constitui um insumo significativo para a análise, desenho e implementação de processos de gestão e mudança.

Palavras-chave: competências em engenharia, educação em engenharia, eficácia institucional, medição da eficácia institucional. 


\section{Introducción}

La formación por competencias de los alumnos universitarios aparece como una tendencia irreversible, casi una exigencia. El objetivo del artículo es reflexionar sobre la concepción actual de la sociedad respecto del egresado universitario - que lo ve como un ser competente, dotado de competencias, capaz de ejercer su profesión en la realidad que lo rodea- y la consecuente necesidad de las universidades $-\mathrm{y}$ la sociedad - de ser efectivas en ese fin y de saber, además, cuán efectivas resultan en cuanto a dotar de competencias a sus profesionales.

El nivel de competencias de sus egresados, evaluado sobre la base de un conjunto de competencias definidas como la "meta", es, en una síntesis simplista, un indicador de la calidad de la formación académica de las universidades. Por ello, es imprescindible, para aquellas universidades que pretendan formar a los profesionales con esta concepción de "ser competente", contar con un conjunto de competencias genéricas de egreso que actúan como la "meta" por alcanzar. Además, es importante que puedan medir su grado de alcance de la meta para poder, en función de esos indicadores, retroalimentar y mejorar sus procesos.

Este artículo presenta el conjunto consensuado de competencias básicas de egreso esperadas para el ingeniero argentino definido por el Consejo Federal de Decanos de Ingeniería de Argentina. Luego, brinda detalles de la primera experiencia de medición de la efectividad institucional en la formación de ingenieros realizada en la Argentina, con base en la autopercepción de los mismos egresados sobre las competencias alcanzadas en el ámbito académico.

Se espera que los lectores puedan analizar las competencias esperadas consensuadas por las facultades de ingeniería de Argentina y ver su aplicabilidad a sus propias realidades; además, que conozcan la experiencia de medición realizada por la Facultad de Ingeniería de la Universidad FASTA y, a partir de ella, puedan diseñar sus propias estrategias de medición.

El trabajo refiere a cuestiones abordadas en un trabajo presentado por los autores en el World Engineering Education Forum (WEEF), 2012.

\section{Marco de referencia}

Nuevos paradigmas como la sociedad del conocimiento, la globalización, las redes y la actual economía conforman un escenario particular que requiere de nuevas formas de intercambio y de comunicación. El mundo cambió y sigue cambiando, y la sociedad actual exige más a la Universidad. No sólo exige la formación profesional -el "saber" - , sino también la dotación de competencias profesionales a sus egresados - el "saber hacer" - . Esto se ve claramente y es asumido así por las universidades a partir de la Declaración de Bolonia de 1999 y la declaración de "la educación como un servicio público" de la Convención de Salamanca de 2001.

El antiguo paradigma de formación de profesionales basado en la enseñanza como simple esquema de transferencia de conocimientos que el alumno oportunamente sabrá abstraer, articular y aplicar con eficacia ha ido perdiendo espacio en la realidad actual. La visión actual de la sociedad propone ver al egresado universitario como un ser competente - con un conjunto de competencias-, capaz de ejercer su profesión en la realidad que lo rodea.

La Comisión Internacional sobre la Educación para el Siglo XxI, convocada por la Unesco y dirigida por el Dr. Jacques Delors, a través de su informe (conocido como Informe Delors) propone que "La educación a lo largo de la vida se basa en cuatro pilares: aprender a conocer, aprender a hacer, aprender a vivir juntos, aprender a ser" [1, p. 34]. Estas cuestiones se transforman en básicas a la hora de pensar, diseñar, implementar y evaluar los procesos de formación en competencias.

En el caso de la ingeniería argentina,

[...] hay consenso en cuanto que el ingeniero no sólo debe saber, sino también saber hacer. El saber hacer no surge de la mera adquisición de conocimientos sino que es el resultado de la puesta en funciones de una compleja estructura de conocimientos, habilidades, destrezas, etc., que requiere ser reconocida expresamente en el proceso de aprendizaje para que la propuesta pedagógica incluya las actividades que permitan su desarrollo [2, p. 10].

Las empresas exigen de los jóvenes graduados un cierto nivel de competencias para el desempeño profesional que no siempre traen consigo. De hecho, muchas sostienen que, necesariamente, deben dedicar los primeros tiempos de la vida laboral del ingeniero en la empresa a que "aprenda a hacer" a partir de los conocimientos que tiene.

Lo cierto es que las universidades tienen la obligación de dotar de ciertas competencias a los graduados - tal vez más de las que dotan hoy-, pero no de gene- 
rar en el graduado competencias que son propias del ejercicio profesional. Instrumentos como las prácticas de laboratorio, ensayos, prácticas profesionales supervisadas, trabajos de campo, proyectos finales en empresas, etcétera, son muy útiles a la hora de formar en competencias, pero no debemos perder de vista hasta dónde llega el compromiso de la universidad y cuánto le corresponde al "ejercicio laboral".

El sólo ejercicio de la profesión va generando nuevas competencias y especialidades en los ingenieros. Tanto la educación formal de posgrado como la informal de actualización profesional permanente, también van generando competencias en el ingeniero. Claramente, estas competencias están fuera de las expectativas de la universidad en lo que hace a carreras de grado de ingeniería. En la formación de grado, las instituciones se proponen desarrollar sólo aquellas competencias que debería poseer el recién graduado y en el nivel de desarrollo adecuado al inicio de su trayecto profesional.

Davini define a la competencia como un "proceso de largo alcance que implica la incorporación explícita e implícita de formas de pensamiento, valoración y actuación en distintos ámbitos sociales". Por otra parte, señala que el desarrollo profesional es aprendizaje continuo, interactivo, acumulativo, que combina varios formatos de aprendizaje [3, p. 111].

En ese proceso continuo, parte debe darse dentro del ámbito académico y parte, en forma permanente e indefinida, en el ejercicio profesional. Es necesario, entonces, marcar esa frontera; decir hasta dónde llega la universidad; fijar el conjunto de competencias que se pretenden lograr en el graduado dentro del ámbito académico.

En un esquema de educación basado en competencias. La explicitación de las competencias buscadas se convierte en "la meta", determina el currículo académico - las materias, los contenidos, las actividades prácticas, la modalidad de dictado de las materias y clases-, el perfil de los docentes, etcétera. La necesidad de formar "seres competentes" respecto de "la meta" hace que el proceso integral de formación deba cambiar, sobre todo, para ser concebido y practicado como un proceso de dotación de competencias al alumno $-\mathrm{y}$, finalmente, al egresado-. Ese es, según parece, el desafío mayor e inevitable de las escuelas de ingeniería por estos días por América Latina. Verdejo sostiene que

Para contar con herramientas y procesos válidos y confiables de evaluación en distintos momentos de la formación, se requiere que la institución tenga previstas estrategias estructurales, de organización, de política institucional y a nivel de programa, coherentes con el enfoque de competencias profesionales [4, p. 172].

En ese contexto, cada universidad, en primer lugar, y la sociedad en general, querrán saber cuán efectivas resultan a la hora de formar ingenieros en competencia. Es necesario, entonces, definir las competencias esperadas en los profesionales y poder medir la efectividad institucional en la formación, es decir, el grado de alcance de tales competencias por parte de los egresados. He ahí una gran oportunidad de investigación y desarrollo de verdadero valor y prácticamente imprescindible en lo que hace a la evaluación de la calidad institucional y la mejora continua de las unidades académicas, donde los egresados - el propio productoretroalimentan y son parte fundamental del proceso.

Para medir efectividad es necesario tener un patrón o referencia. En este sentido, los decanos de las facultades de ingeniería de la Argentina, reunidos en el seno de su asociación nacional, el Consejo Federal de Decanos de Ingeniería de Argentina (en adelante, CONFEDI), sintetizaron y acordaron las 10 competencias básicas y genéricas deseables para cualquier ingeniero argentino. Esas competencias son, en definitiva, "la meta" por alcanzar por las facultades de ingeniería de Argentina a la hora de dotar de competencias a sus ingenieros.

Luego, a partir de estas competencias básicas consensuadas a nivel nacional, un grupo de investigación interdisciplinario ${ }^{1}$, integrado por profesionales de ingeniería y de ciencias de la educación de la Universidad FASTA, llevó adelante un proyecto de investigación inédito, para determinar el grado de alcance de esas competencias esperadas en el universo de los egresados de la Facultad de Ingeniería de esa universidad.

La investigación realizada (en adelante "la investigación”) significó el estudio de la percepción de los egresados de una unidad académica - la muestra incluyó al 48\% de los egresados- respecto de las competencias adquiridas en su formación.

1 El equipo de investigación estuvo dirigido por la Dra. Mónica Prieto e integrado por los siguientes investigadores: Ing. Agustina Barilari, Ing. Matías Calvo, Lic. Sandra Cirimelo, Lic. Valeria Fernández y Lic. Silvana Sandez. 


\section{Competencias}

Perrenoud propone la siguiente noción de competencia:

Una competencia permite hacer frente regular y adecuadamente, a un conjunto o familia de tareas y de situaciones, recurriendo a las nociones, a los conocimientos, a las informaciones, a los procedimientos, los métodos, las técnicas y también a las otras competencias más específicas [5, p. 3].

Le Bofert asimila las competencias a un saber movilizar:

Poseer conocimientos o capacidades no significa ser competente. Podemos conocer las técnicas o las reglas de gestión contable y no saberlas aplicar en un momento oportuno. Podemos conocer el derecho comercial y redactar mal los contratos. Cada día, la experiencia muestra que las personas que están en posesión de conocimientos o de capacidades no las saben movilizar de forma pertinente y en el momento oportuno, en una situación de trabajo. La actualización de lo que se sabe en un contexto singular (marcado por las relaciones de trabajo, una cultura institucional, el azar, obligaciones temporales, recursos...) es reveladora del "paso (pasaje)" a la competencia. Ella se realiza en la acción [6].

En este sentido, se concibe a la competencia como una actuación o desempeño que debe lograr el estudiante - al poseer ciertos conocimientos básicos- y auxiliado, muchas veces, por el docente, pero que finalmente deberá aprender a movilizar por sí mismo, de acuerdo con los requerimientos propios de su profesión, con sentido de oportunidad.

CONFEDI confirma que muchas de las competencias que se propugnan son destrezas-facetas. Esta definición supone entonces que se alude a capacidades complejas e integradas, relacionadas con saberes (teórico, contextual y procedimental), que se vinculan con el saber hacer (formalizado, empírico, relacional), que están referidas al contexto profesional - entendido como la situación en que el profesional debe desempeñarse o ejercer-, apuntan al desempeño profesional que se pretende - entendido como la manera en que actúa un profesional técnicamente competente y socialmente comprometido-y que permiten incorporar la ética y los valores [2].

\subsection{Competencias de Ingeniería}

“Qué implica una enseñanza orientada al desarrollo de competencias en ingeniería?” [2]

Para favorecer el desarrollo de competencias, se requiere tener claridad sobre cuáles son las competencias que deben ser consideradas en todos los estudios de ingeniería. Ello supone pensar la formación de grado del ingeniero desde el eje de la profesión, es decir, desde el desempeño, desde lo que el ingeniero efectivamente debe ser capaz de hacer en los diferentes ámbitos de su quehacer profesional y social en sus primeros años de actuación profesional.

Según Brailovsky [7], la "competencia" es un constructo sólo accesible de manera parcial, a través de aspectos que se pueden medir, como los conocimientos, las habilidades técnicas, las actitudes, la capacidad de solucionar problemas, el razonamiento, la capacidad de comunicar a otros, etcétera.

El CONFEDI hace algunas apreciaciones respecto de las competencias que explican los diversos "niveles de dominio" de las competencias a lo largo de la trayectoria académica. También se plantea la cuestión de la necesaria formación integral, que va más allá de la formación técnica, fundamental para tener en cuenta a la hora de evaluar.

\subsection{Delimitación de la formación de grado}

Para la delimitación de la formación de grado, desde el punto de vista de la formación en competencias, es fundamental tener en cuenta dos cosas:

a) Diferenciar las competencias de egreso de las competencias profesionales. Las competencias de egreso se desarrollan a través de las prácticas preprofesionales realizadas por los estudiantes a lo largo del trayecto curricular compuestos por sus estudios académicos, orientadas a capacitarlos para una efectiva inserción laboral. Esto implica un determinado nivel de desarrollo o grado de dominio de las competencias del recién graduado. En contraposición, las competencias profesionales terminan de desarrollarse a través de las prácticas profesionales que el ingeniero realiza durante el ejercicio de su profesión a lo largo de varios años, y suponen, por tanto, un nivel de desarrollo o grado de dominio superior al anterior. 
Desde el punto de vista del desarrollo de competencias, el propósito formativo del plan de estudios de la carrera debe estar definido por el perfil de competencias de egreso, condición necesaria para la inserción laboral del recién graduado. Dado que las competencias de egreso se refieren al recién graduado, se busca un grado de desarrollo de las mismas adecuado aunque no óptimo - pues ello requeriría de la experiencia laboral-, razón por la cual la adquisición del nivel establecido puede ser alcanzado aun cuando no se hayan adquirido todas las capacidades implicadas.

b) Reconocer la relación existente entre las actividades reservadas al título y las competencias de egreso: las actividades reservadas al título definen aquellas actividades que legalmente están reservadas para los profesionales con una determinada titulación. Este marco es más abarcador, pues cubre cualquier actividad profesional. De ninguna manera implica que el recién graduado deberá estar capacitado para realizar competentemente cualquiera de ellas. Esto es fundamental tenerlo presente al momento de definir el perfil de competencias de egreso, para no sobrecargar de contenidos los planes de estudios. Por otro lado, las competencias de egreso no deben estar limitadas sólo al marco de las actividades reservadas al título, ya que la formación académica debe ser de naturaleza integral y no solamente técnica.

Estas nociones sirven de referencia para determinar la calidad del proyecto académico y el recorte que se hace en este proyecto respecto de las características de la población seleccionada para analizar las competencias de los graduados de Ingeniería.

\subsection{Clasificación de las competencias de egreso en ingeniería [2]}

Existen diversas clasificaciones de competencias realizadas por expertos, basadas en criterios también diversos. Lo complejo de la cuestión radica en que no hay categorías unívocas.

Por ejemplo, el modelo de competencias profesionales integrales planteado por Huerta Amezola y colaboradores [8] establece tres niveles: las competencias básicas - capacidades intelectuales indispensables para el aprendizaje de una profesión; en ellas se encuentran las competencias cognitivas, técnicas y metodológicas, muchas adquiridas en los niveles edu- cativos previos-, las genéricas - son la base común de la profesión o se refieren a las situaciones concretas de la práctica profesional que requieren de respuestas complejas- y las específicas - con la base particular del ejercicio profesional vinculadas a condiciones específicas de ejecución.

Otros estudios, como el Proyecto Mecesup (Programa de Mejoramiento de la Calidad y la Equidad de la Educación Superior) organizan las competencias en cuatro grandes ejes: generales, transversales, técnicas específicas y las de saber fundante [9].

$\mathrm{Al}$ referirse a "competencias genéricas" en este artículo, se hará alusión a aquellos comportamientos que son más universales, en este caso, todas aquellas acciones que tienen que ver con la actividad profesional de un ingeniero. Al hablar de competencias "específicas", se hará referencia a la actividad que debe desempeñar un ingeniero, según su especialidad. Este ha sido el criterio acordado por los miembros del CONFEDI para la República Argentina, que distingue:

Competencias genéricas: se adopta un planteo abarcativo de competencias genéricas. Se considera como tales, a aquellas competencias profesionales comunes a todos los ingenieros.

Competencias específicas: son las competencias profesionales comunes a los ingenieros de una misma "terminalidad" (por ejemplo: Ingeniería Ambiental, Ingeniería en Informática, Ingeniería Industrial, Ingeniería Electrónica, etc.).

\subsection{Desagregación de una competencia en capacidades [2]}

Para la propuesta de competencias, CONFEDI consideró que no era conveniente plantear una sumatoria extensa de competencias demasiado detalladas, debido a que las competencias son capacidades integradas y complejas, por lo cual es pertinente un abordaje sintético desde la complejidad, que luego se desagregue en niveles componentes adecuados para una implementación curricular.

Por esto se adoptó un esquema con diez competencias genéricas de la ingeniería, desagregadas en dos niveles simples e integradores de capacidades.

Una competencia requiere la articulación eficaz de diversas capacidades, expresadas como "ser capaz de.... 


\section{Competencias genéricas de egreso en las carreras de ingeniería}

El CONFEDI ha realizado una minuciosa selección de Competencias Genéricas que considera fundamentales en la formación de cualquier graduado de Ingeniería en la República Argentina. Estas son [2]:

\section{Competencias tecnológicas}

1. Competencia para identificar, formular y resolver problemas de ingeniería

2. Competencia para concebir, diseñar y desarrollar proyectos de ingeniería (sistemas, componentes, productos o procesos)

3. Competencia para gestionar - planificar, ejecutar y controlar- proyectos de ingeniería (sistemas, componentes, productos o procesos)

4. Competencia para utilizar de manera efectiva las técnicas y herramientas de la ingeniería

5. Competencia para contribuir a la generación de desarrollos tecnológicos o innovaciones tecnológicas.

Competencias sociales, políticas y actitudinales

6. Competencia para desempeñarse de manera efectiva en equipos de trabajo

7. Competencia para comunicarse con efectividad

8. Competencia para actuar con ética, responsabilidad profesional y compromiso social, considerando el impacto económico, social y ambiental de su actividad en el contexto local y global ${ }^{2}$

9. Competencia para aprender en forma continua y autónoma

10. Competencia para actuar con espíritu emprendedor.

Cabe señalar que se parte del supuesto de que las competencias de egreso no se identifican exactamente con las denominadas "competencias profesionales", ya que estas últimas terminan de desarrollarse a través del ejercicio profesional (tal como sugiere también el CONFEDI).

También es importante señalar que sólo es posible analizar la autopercepción de cada una de las competencias planteadas por el CONFEDI que poseen los

2 Es importante destacar la importancia de la competencia 8 en el marco de esta revista que propone una ingeniería solidaria. Si bien la solidaridad es un valor, la competencia para actuar con ética, responsabilidad profesional y compromiso social, considerando el impacto económico, social y ambiental de su actividad en el contexto local y global, es determinante de actitudes solidarias. graduados, en relación con las expectativas que estos poseen respecto del trabajo realizado para alcanzar sus metas, y en relación con el nivel de desarrollo de las competencias percibido para la realización de cada una de las tareas sugeridas.

\section{La experiencia de medición de las competencias}

Sobre la base de las competencias "meta", surge en la Facultad de Ingeniería de la Universidad FAsTA la inquietud de medir su "eficacia institucional". Para ello se diseña un proyecto de investigación de corte evaluativo, esto es, que centra su interés en conocer el nivel de consecución de alguna meta u objetivo existente. En este caso se trata de analizar el valor del currículum en cuanto a los resultados obtenidos por los graduados de carreras de Ingeniería de la Universidad FASTA.

Para ello se elaboró una encuesta "autoadministrada", con preguntas referidas a las competencias propuestas por el CONFEDI para los graduados de todas las carreras de la Facultad de Ingeniería. La encuesta realizada consta de más de 30 preguntas, varias de ellas con múltiples opciones de respuesta, utilizando escalas de tipo Likert. La misma se aplicó y completó sólo por vía web.

Para la investigación se convocó a los graduados de las carreras de Ingeniería Ambiental e Ingeniería Informática de la Facultad de Ingeniería de la Universidad FASTA entre el 2006 y 2010, obteniendo una muestra relevada de 104 individuos, determinada por la respuesta voluntaria de estos.

Para validar el instrumento principal (encuesta) en cuanto a su contenido y como constructo, se tomó como base la selección de las diez competencias genéricas elaborada por CONFEDI para todas las carreras de ingeniería del país. Dentro de cada una de las competencias genéricas se enumeran distintas capacidades que desglosan, en cierto modo, el amplio espectro de cada competencia. Se incluyeron algunas de estas capacidades para visualizar si la competencia era reconocida por el graduado o no. Algunas de las capacidades relativas a otras competencias fueron utilizadas como distractores.

El $80 \%$ de los ítems de la encuesta utilizados para medir el grado en que los egresados han alcanzado las competencias responden a escalas de Likert. El instrumento fue validado por el coeficiente Alfa de Cronbach de consistencia interna, que alcanzó un valor de 0,768. 
La encuesta desarrolla sus 30 ítems directamente relacionados con las diez competencias genéricas que presenta el CONFEDI.

La participación en la encuesta fue anónima y por lo tanto se tomaron todos los recaudos necesarios para mantener dicha condición y también la privacidad y seguridad de los datos, tanto de los datos personales de los egresados, como los resultados obtenidos por la institución en el estudio.

Para garantizar el anonimato y la privacidad de los participantes, pero al mismo tiempo permitir un mecanismo de seguimiento que posibilite saber en todo momento quiénes habían respondido a la encuesta y quiénes no, se generaron códigos de acceso aleatorios únicos para cada usuario. Las respuestas fueron almacenadas por código, sin relación con el usuario.

El sistema web utilizado para la administración de la encuesta fue desarrollado "a medida" basado en un software open source existente. Dicho sistema se programó en lenguaje PHP y base de datos en MySQL.

Las invitaciones y recordatorios se distribuyeron por correo electrónico, incluyendo una breve descripción del proyecto y sus objetivos, y una dirección web de acceso a la encuesta.

Todos los datos obtenidos en la encuesta se trabajaron estadísticamente con el programa XLStat.

Dado que la investigación trató de "medir la eficacia académica" que está referida a procesos de evaluación de calidad institucional, donde los egresados forman parte de los aspectos por evaluar - en la medida que constituyen el producto central de la tarea universitaria-, es necesario considerar que tal evaluación se realizó exclusivamente desde la óptica de los propios graduados, a partir de una "autoevaluación". Es fundamental contemplar esta cuestión, ya que en la autoevaluación entran en juego variables como la propia percepción de la eficacia académica, que podrían incidir directamente sobre los resultados obtenidos. Para la autoevaluación de las competencias, los graduados ponen en juego elementos como valores, concepciones de aprendizaje, etcétera. Habitualmente, la intervención de estos factores no siempre es consciente para quienes se autoevalúan.

\section{La eficacia institucional}

Para los decanos, secretarios académicos, directores de carreras y gestores de la educación superior en general, es fundamental lograr la eficacia de sus carreras.
Eso es gestión universitaria. La eficacia es "el desafío" de la gestión, dado que es un indicador clave del éxito de las carreras y unidad académica. La gestión hace al proceso administrado y controlado de búsqueda de esa eficacia en un marco de excelencia académica.

Todos y cada uno de los procesos y las actividades de la gestión universitaria, desde la planificación estratégica hasta la administración operativa cotidiana, deberían estar alineadas tras la búsqueda de la eficacia, y cada persona dentro de la institución debería conocer qué aporta, desde su lugar, a la eficacia de las carreras y unidad académica.

Pero lograr la eficacia no es sólo una cuestión exclusiva de la gestión. Es una cuestión de todos los miembros de la comunidad académica y "de tiempo completo". La docencia, investigación y extensión, como funciones universitarias básicas, más la integración a la sociedad, la vinculación y transferencia tecnológica, los convenios y proyectos con terceros deben, también, ayudar a alcanzar la eficacia. Los procesos de revisión, cambio e implementación de planes de estudio deben estar, indefectiblemente, conducidos y analizados desde la perspectiva de las competencias buscadas de egreso y el perfil profesional del graduado.

Por eso, "pensar y actuar" en cuanto a competencias significa un cambio radical en los fines y medios académicos tradicionales. Buscar permanentemente y alcanzar la eficacia respecto a competencias en una unidad académica aparece, en el corto y mediano plazo, como la gran revolución metodológica de las instituciones y comunidades académicas.

Es imposible mejorar sin medir. Sólo la medición confiable, sistemática y permanente permite mejorar en forma continua. En este contexto, la medición de las competencias de los graduados de una unidad académica es un instrumento clave para generar información que retroalimente a esa unidad académica, y sólo a esa, y la haga reflexionar al respecto; luego, le permite diseñar estrategias de intervención e intervenir; por último, le permite evaluar el impacto de la intervención y verificar la mejora.

Seneca decía: "no hay vientos favorables para quien no sabe a dónde va". Si el necesario y permanente proceso de revisión y ajuste de los planes de estudios se hace guiado por un conjunto de competencias para alcanzar, claras y consensuadas, es más sencillo, pues sabemos a dónde queremos llegar. Si no tenemos en cuenta las competencias, el diseño de una carrera se transforma en un desafío de organización de contenidos en una grilla estática con cierta coherencia en 
cuanto a su correlación. Son los contenidos, los que mandan... casi lo único que importa. El objetivo es ver cómo organizamos todos los contenidos que el profesional debe conocer o saber. Cuando uno trabaja en el diseño de una carrera con base en competencias, necesita una meta que incorpora en el proceso una serie de "otras dimensiones" para tener en cuenta —más allá de los contenidos-y que deben articularse todas para lograr el "producto profesional" buscado, no sólo en cuanto a lo que "sabe" o aprendió -o sólo recibió...-, sino también en lo que "es" y lo que "sabe hacer". Cuando hablamos de competencias, la discusión no es tan sencilla como "si ponemos tal o cual contenido en la carrera”. La discusión parte de “iqué deberíamos hacer para que el graduado sea capaz de...?", “itiene sentido ese contenido?”, “¿para qué competencias es necesario ese contenido?", “¿cómo deberíamos transmitir ese contenido a los alumnos para que efectivamente contribuya a las competencias buscadas?" "¿cómo el alumno, a partir de ese contenido, aprende a hacer algo?" La discusión va más allá del "qué". Incluye, indefectiblemente, el "cómo", pensando siempre en el "porqué".

Resuelto esto, como proceso a la hora de modificar una carrera es fundamental saber dónde estamos, para saber qué debemos mejorar. Si no estamos dispuestos a cambiar, no podremos mejorar. Si no sabemos qué debilidades tenemos, tampoco podremos mejorar. Siguiendo con la idea de Séneca, podríamos decir que "tampoco hay vientos favorables para quien no sabe dónde está...”. He ahí la necesidad de medir y conocer nuestro producto, si pretendemos mejorarlo. Esa es la invitación.

En síntesis, saber medir y poder medir la eficacia de una carrera o unidad académica es una condición necesaria - aunque no suficiente- para el diagnóstico, $\mathrm{y}$ un factor determinante de la capacidad institucional para mejorar. De eso trata esta experiencia.

Volviendo a la investigación, no se presentan aquí resultados de esta, haciendo hincapié en que lo valioso de la experiencia es la investigación misma, su proceso, análisis y diseño metodológico que son, en definitiva, el verdadero aporte a la gestión universitaria, en particular, en el ámbito de la ingeniería. Los resultados de la investigación sólo tienen valor para la institución que mide su eficacia.

Sólo se pretende compartir la experiencia con otras instituciones y gestores universitarios para motivarlos a la reflexión y debate sobre el tema y a la reproducción de esta en otras instituciones. Los resultados y detalles de la investigación pueden ser solicitados a los autores.

No obstante, cabe señalar que los indicadores obtenidos fueron muy satisfactorios respecto de la eficacia institucional en función de las competencias de egreso adquiridas por los graduados.

Además, del análisis de los resultados de las competencias en forma individual se realizó un análisis de los datos obtenidos respecto de las competencias en su conjunto.

\section{Conclusiones}

El análisis del grado de percepción promedio del nivel de desarrollo de cada competencia, el desagregado de los ítems que lo determinan y el de las competencias en su conjunto contribuyen al análisis y diagnóstico institucional, fundamentalmente desde lo curricular. La percepción del grado de adquisición de las competencias por parte de los graduados es un elemento útil para medir la eficacia institucional.

La Facultad de Ingeniería de la Universidad FasTA pudo diseñar e implementar un sistema de medición de las competencias, a partir de la autopercepción de los propios graduados; pero ese es sólo un elemento indicativo, y no debe ser el único para considerar. Otros estudios que brinden elementos de complemento y validación, tales como la medición de la percepción de los empleadores de los egresados respecto de las competencias demostradas en el ejercicio de la profesión, deben ser realizados, para una visión multifocal de la situación.

La toma de decisiones en la gestión académica de las carreras de ingeniería requiere de insumos confiables y adecuados a la realidad propia de cada institución, si se pretende efectividad en los resultados, en este caso, en la dotación de competencias en los graduados. En tal sentido, el trabajo interdisciplinario entre ingenieros y profesionales de ciencias de la educación es clave en este tipo de investigaciones y debe ser incorporado como parte fundamental del proceso de gestión académica.

Si el desafío de las escuelas de ingeniería pasa por la necesidad de formar "seres competentes" respecto de "la meta", y eso requiere cambiar el modelo actual de formación por uno orientado a la dotación de competencias, este tipo de investigaciones son un aporte significativo para el análisis, diseño e implementación de tal proceso de cambio. 
El marco teórico, el análisis de la investigación sobre las competencias en general y su aplicación a las definidas por CONFEDI, el diseño metodológico y el instrumento diseñado y aplicado son aportes valiosos de esta investigación a la comunidad de gestión académica, sobre todo por pionera.

Disponer en Argentina de la explicitación de las competencias genéricas de egreso para profesionales de ingeniería, consensuada por todas las facultades de ingeniería del país, es un aspecto esencial a la hora de considerar y de medir la eficacia institucional a la luz de tales competencias.

\section{Agradecimientos}

Los autores hacen público su agradecimiento al equipo del proyecto de investigación en Competencias en Ingeniería de la Universidad FASTA, dirigido por la Dra. Mónica Prieto. También a la Secretaria de Investigación de la Facultad de Ingeniería, Lic. Mónica Pascual, por su colaboración.

También agradecen a la revista Ingeniería Solidaria la invitación para compartir su parecer con colegas de Colombia y otros países.

\section{Referencias}

[1] J. Delors, La educación encierra un tesoro. Informe a la Unesco de la Comisión Internacional sobre la educación para el siglo XXI, Madrid: Santillana-Ediciones Unesco, 1996.

[2] Consejo Federal de Decanos de Ingeniería (CONFEDI), Competencias Genéricas. Desarrollo de competencias en la Enseñanza de la Ingeniería Argentina. San Juan, Argentina: Universidad Nacional de San Juan, Argentina, 2007.

[3] M. C. Davini, La formación docente en cuestión. Políticas y pedagogía, Paidós: Buenos Aires, 1995.

[4] P. Verdejo, Modelo para la educación y evaluación por Competencias (MECO). Proyecto $6 \times 4$ UEALC, informe final, 2008.

[5] Ph. Perrenoud, "Construir las competencias, ¿es darle la espalda a los saberes?". Red U. Revista de Docencia Universitaria, número monográfico II Formación centrada en competencias (II), 2008.

[6] G. Le Bofert, De la competence. Essai sur un attracteur étrange, Paris: Les Éditions dòrganisation, 1994.

[7] C. Brailovsky, "Educación médica, evaluación de competencias", en ops/oms (eds.), Aportes para un cambio curricular en Argentina, Buenos Aires; University Press, 2001.

[8] J. Huerta Amezola, I. Pérez García, y A. Castellanos Castellanos, "Desarrollo curricular por competencias profesionales integrales”. Revista EDUCAR, No. 13, 2000. [En línea]. Disponible en: http://www2.ufro.cl/docencia/documentos/Competencias.pdf.

[9] Programa de Mejoramiento de la Calidad y la Equidad de la Educación Superior en Chile, Ministerio de Educación de Chile, Informe final de evaluación, Mecesup, 2004. [En línea]. Disponible en: http://www.mecesup. uchile.cl/acerca.html 